\title{
How Effective Are Antioxidant Supplements in Obesity and Diabetes?
}

\author{
Daniyal Abdali Sue E. Samson Ashok Kumar Grover \\ Department of Medicine, HSC 4N41, McMaster University, Hamilton, Ont., Canada
}

\author{
Key Words \\ Vitamin C. Green tea - Green coffee · Cinnamon - Lipoic \\ acid. Carnitine
}

\begin{abstract}
Obesity is a central health issue due to its epidemic prevalence and its association with type 2 diabetes and other comorbidities. Obesity is not just being overweight. It is a metabolic disorder due to the accumulation of excess dietary calories into visceral fat and the release of high concentrations of free fatty acids into various organs. It represents a state of chronic oxidative stress and low-grade inflammation whose intermediary molecules may include leptin, adiponectin and cytokines. It may progress to hyperglycemia, leading to type 2 diabetes. Whether or not dietary antioxidant supplements are useful in the management of obesity and type 2 diabetes is discussed in this review. Only the benefits for obesity and diabetes are examined here. Other health benefits of antioxidants are not considered. There are difficulties in comparing studies in this field because they differ in the time frame, participants' ethnicity, administration of antioxidant supplements, and even in how obesity was measured. However, the literature presents reasonable evidence for marginal benefits of supplementation with zinc, lipoic acid, carnitine, cinnamon, green tea, and possibly vitamin $C$ plus $E$, although the evidence is much weaker for omega -3 polyunsaturated fatty acids, coenzyme Q10, green
\end{abstract}

coffee, resveratrol, or lycopene. Overall, antioxidant supplements are not a panacea to compensate for a fast-food and video-game way of living, but antioxidant-rich foods are recommended as part of the lifestyle. Such antioxidant foods are commonly available.

(c) 2015 S. Karger AG, Basel

\section{Introduction}

Obesity and the associated comorbidities such as diabetes and cardiac diseases are lead causes of human morbidity and mortality. The epidemic prevalence of obesity has been associated by some with fast foods and a sedentary lifestyle [1]. The treatment of obesity is a mammoth industry, but the biological mechanism of this disease is not understood. Programs such as the Kuwait National Programme for Healthy Living are being developed to promote the health and well-being of individuals [1]. One of the hypotheses describes obesity as a low-grade inflammation with systemic changes caused by the accumulation of visceral fat [2]. It is interesting that antioxidants have been suggested to ameliorate the ailments due to inflammation. The biology of obesity and the associated inflammatory molecules are examined here first, and then the literature is reviewed to test the hypothesis that antioxidant supplements are beneficial in the management of obesity and/or type 2 diabetes. 


\section{What Is Obesity}

\section{Definition and Diagnosis of Obesity}

The World Health Organization (WHO) defines obesity as a condition of abnormal or excessive fat accumulation in adipose tissue such that health may be impaired [3]. Clinically, in adults, obesity is defined as having a body mass index (BMI) greater than 30 , specifically, with an abnormal fat distribution. However, this definition is inadequate since it does not take into account the regional distribution of the fat within the body - abdominal/ visceral versus subcutaneous. The abnormal distribution of fat is a risk factor for obesity and its comorbidities.

Obesity accompanies major systemic changes in the body. Abdominal (or visceral) obesity is associated with an elevated outflow of free fatty acids (FFA) from the visceral fat depots and metabolic dysregulation, including insulin resistance [4]. Hypertrophied intra-abdominal adipocytes may undergo hyperlipolysis, leading to an increased flow of FFA to various organs, including the liver. The increase in FFA flow may impair liver function, leading to increased hepatic glucose production and insulin resistance. The hepatic insulin resistance is associated with a decreased apolipoprotein B degradation and an increased production of lipoproteins rich in triglycerides (TG) [5]. In obese patients, there is an infiltration of macrophages into adipose tissue, resulting in a chronic lowgrade type of inflammation. Other factors that may contribute to the altered metabolic profile of obese patients include proinflammatory molecules such as interleukin (IL)- 6 and tumor necrosis factor- $\alpha$ (TNF- $\alpha$ ). A confirmation of the inflammatory status of visceral obesity is an increase in the inflammation markers such as the plasma levels of C-reactive protein (CRP) [5]. Obesity also involves changes in other molecules such as leptin, adiponectin and endothelium adhesion molecules. Another probable explanation is that excess intra-abdominal fat is a marker of the inability of subcutaneous adipose tissue to store the excess energy. This inability of the subcutaneous tissue to hold onto the extra fat, known as ectopic fat deposition, results in the excess fat being stored at undesired sites such as the liver, the skeletal muscle and the heart, as well as in pancreatic $\beta$ cells [5].

The increased force of the fat mass leads to difficulties in locomotion and pain in the back, hip, knee, ankle, or foot. Furthermore, the joints, tendons, fascia, and cartilage are all affected because they have to compensate for the increased weight on the knee joint. The weight-bearing joints may subsequently develop osteoarthritis. Insulin resistance and metabolic syndrome increase the risk factor for cardiovascular diseases [6]. Circulating FFA together with other factors may lead to increased levels of low-density lipoprotein (LDL), which in turn would increase the size of atherosclerotic plaques. Plaques can lead to thrombosis and myocardial infarctions. Excess soft tissue and fat in the chest wall decreases respiratory muscle compliance, functional residual capacity and total volume, which leads to a mismatch in ventilation-perfusion [6]. It has been suggested that obstructive sleep apnea may be in the causal pathway between obesity and asthma [6]. Obesity may also increase the risk factor for certain types of cancer. Diabetes associated with obesity has the highest morbidity and mortality burden and will be the focus of this review.

\section{Physical Diagnostic Measures}

The crudest, simplest and commonest way of measuring obesity is the BMI. A BMI greater than 30 is the broad clinical diagnosis for obesity. However, its first major pitfall is that it does not indicate the type and the locale of the fat in the body. Individuals with excess fat stores within their abdominal regions (as determined by a waist circumference measurement) are at particular risk of the adverse health consequences resulting from obesity. Also, BMI may vary with age, gender and ethnicity. Particularly, BMI would vary considerably during early growth [3]. Thus, at its best the BMI can be used as a flagging factor but not for the diagnosis of obesity. Even waist circumference may be a better measure of obesity than BMI because it is better associated with abdominal fat. A study of the Middle East population determined the waist-tohip ratio to be a better measure of comorbidities associated with obesity [7].

Techniques currently available for investigating fat distribution include water displacement, dual-energy Xray absorptiometry, computerized tomography and magnetic resonance imaging. Water displacement is considered the gold standard in total body fat measurement, but it is time-consuming, inconvenient, not widely available and, most importantly, it does not convey any information about body fat distribution [8]. Dual-energy X-ray absorptiometry is a relatively accurate test. However, the major downside to this test is the exposure to high doses of ionizing radiation. Computerized tomography is quick and suitable for automated image analysis and reproducible. However, the radiation makes repeated testing prohibitive. Finally, magnetic resonance imaging does not involve any ionizing radiation exposure. Its accuracy and reproducibility for fat analysis has been validated with cadaveric dissections and through animal models. A clear 
representation of fat distribution can be obtained with minimal inaccuracies by combining magnetic resonance imaging with computer-assisted diagnostic techniques. This combined method makes it possible to conduct body fat distribution analysis on a daily basis with relative ease, efficiency and effectiveness [8]. However, the snag is that these tools are expensive and hence not readily available.

\section{Biochemical and Genetic Diagnostic Measures}

Variants in the following three genes have been associated with the risk of obesity: (1) the FTO gene was found (through a genome-wide association study) to be associated with type 2 diabetes and was further determined to be an obesity gene; (2) a common variant in the MC4R gene was seen to be associated with BMI and, as an extension, obesity, and (3) the PCSK1 gene is associated with obesity. Current evidence suggests a hypothalamic role for all three genes [9]. Overall, the evidence for genetic intervention or screening is too immature. There are large gaps in knowledge, and the findings of the various studies are ethnically dependent. Furthermore, many variants of these genes are not associated with obesity or type 2 diabetes. Thus, genetic screening is not a viable option until more is known in this field.

The screening for obesity may also involve monitoring of metabolic disorders. The five screening variables used to identify those with metabolic syndrome, as proposed by the National Cholesterol Education Program-Adult Treatment Panel III, are waist circumference, circulating levels of TG and high-density lipoprotein (HDL), cholesterol, fasting blood glucose (FBG), and blood pressure. The presence of three or more of the following criteria is defined as metabolic syndrome: blood pressure $\geq 130 / 85$ $\mathrm{mm} \mathrm{Hg}$, waist circumference $>102 \mathrm{~cm}$ in men and $>88 \mathrm{~cm}$ in women, HDL cholesterol $<1.036 \mathrm{mM}(40 \mathrm{mg} / \mathrm{dl})$ in men and $<1.295 \mathrm{mM}(50 \mathrm{mg} / \mathrm{dl})$ in women, $\mathrm{TG} \geq 1.695$ $\mathrm{mM}(150 \mathrm{mg} / \mathrm{dl})$, and FBG $\geq 6.1 \mathrm{~mm}(110 \mathrm{mg} / \mathrm{dl})$ [5]. Note that these criteria combine a physical diagnostic measure with the biochemical ones.

\section{Obesity and Type 2 Diabetes}

Type 1 diabetes, or juvenile diabetes, is due to autoimmune destruction of pancreatic $\beta$ cells which are normally responsible for producing the hormone insulin. The patient has to be given insulin on a regular basis. In type 2 diabetes, insulin is either not produced in sufficient amounts or does not elicit the normal response from cells. Insulin is needed to supply glucose to the cells of the heart, skeletal muscle and adipose tissue, mostly via the facultative transporter GLUT4, although low levels of GLUT1 are also present in these cells $[10,11]$. Insulin stimulates the translocation of GLUT4-containing vesicles from intracellular stores to the plasma membrane. This results in an immediate 10 - to 20 -fold increase in glucose transport [10]. Furthermore, the defect seen in type 2 diabetes may also be a result of impairments in the translocation machinery, as defined by molecules involved in GLUT4 sorting, retention, movement, docking and tethering, and fusion [11]. In type 2 diabetes, the insulin-dependent increase in the level of surface GLUT4 is defective. Though insulin is not necessary for glucose uptake via the transporters GLUT1-3, GLUT4 transporters are essential for lowering the acute postprandial rise of plasma glucose levels via sensitivity to insulin levels. Furthermore, since skeletal muscles and adipose tissue are the largest storage areas for glucose, and the GLUT4 transporter is found within them only, the importance of insulin in controlling blood sugar levels after a meal cannot be overstated [10]. Insulin defects lead to very high plasma levels of glucose which can damage various organs. Type 2 diabetes may or may not be related to obesity. The management regimens involve mainly a reduction of blood glucose levels using diet and exercise for weight control, drugs which prevent conversion of other metabolites into glucose and, if needed, insulin therapy.

Without the proper adipose tissue precursors, the fatty acid storage decreases and the amount of FFA in circulation increases (lipotoxicity). Excess adiposity due to a positive caloric balance leads to the release of inflammatory molecules (from central visceral fat depots) that lead to insulin resistance and increased concentration of circulating FFA. This cycle keeps perpetuating itself and the increasing amounts of FFA are continuously deposited in muscles and liver (fig. 1) [6].

The pathophysiology of obesity cannot be explained by insulin resistance alone. Adipose tissue is an organ of energy storage and also actively participates in hormonal regulation of homeostatic systems. Adipose tissue can be distinguished into two major types: brown and white adipose tissue [12]. The brown adipose tissue serves as a source of thermogenesis during periods of nonshivering. The white adipose tissue is the majority of adipose tissue in the body and is the site of energy storage. Approximately $10 \%$ of the white adipose tissue is composed of macrophages. The number of macrophages present is correlated with the amount of adiposity and the size of the adipocytes. 
Fig. 1. Pathways from excess calorie consumption to obesity and diabetes. Excess calories lead to blockade of the steps in the pathways, as shown by ' $\mathrm{x}$ '.

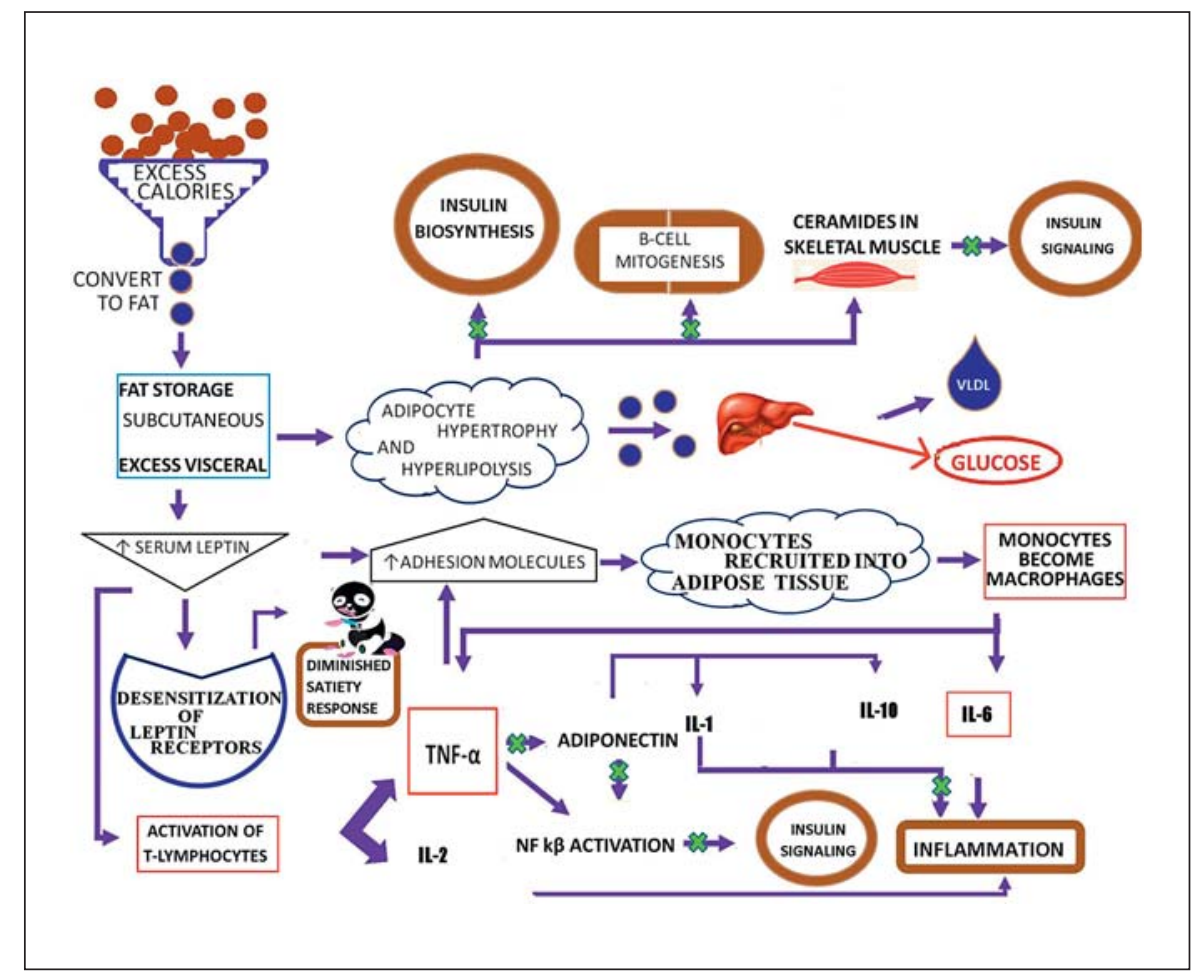

The adipose tissue is also the source of a number of modulatory molecules. Leptin, a satiety hormone, is a $16-\mathrm{kDa}$ protein produced mainly by adipocytes [12]. It is hypothesized that obese individuals may become desensitized to leptin, and thus even if leptin levels remain high, they may not feel satisfied after eating. Leptin also regulates inflammation through $\mathrm{T}$-cell proliferation and activation, and by protecting $\mathrm{T}$ lymphocytes from apoptosis. Leptin influences $\mathrm{T}$ lymphocytes to release cytokines such as IL-2 and TNF- $\alpha$, a Th1 response that activates the innate immune system involving IL- 6 and TNF- $\alpha$ [12]. TNF- $\alpha$ is a pivotal molecule in obesity-mediated type 2 diabetes [13]. It may cause insulin resistance directly by increasing serine phosphorylation of the insulin receptor. Adiponectin is best known for its role in mediating insulin sensitivity. Adiponectin is released mostly by adipocytes [12]. Unlike leptin levels, which increase in response to increased adiposity, adiponectin levels decrease. Adhesion molecules on endothelium are upregulated by leptin, along with other molecules produced by adipocytes. The increased expression of adhesion molecules leads to an increased transmigration of bone marrow-derived monocytes, thus leading to an increase in macrophages residing in the white adipocyte tissue. Some of these macrophages fuse and create giant multinucleated cells. These macrophages produce higher amounts of TNF- $\alpha$, IL- 6 and chemokines compared to those in lean individuals [12].

The probable mechanisms of obesity and diabetes are summarized below and in figure 1 :

- The daily caloric intake exceeds the daily caloric output.

- The excess calories are converted to fat and initially stored in subcutaneous fat stores.

- As the subcutaneous fat stores fill to capacity, fat is stored in the visceral fat stores.

- As the adipose mass starts to increase, there is a concurrent increase in leptin levels and, thus, an increase in the adhesion molecules on endothelial cells.

- The increased concentrations of serum leptin lead to the following: (1) desensitization of leptin receptors in the hypothalamus - thus, even though the fat stores are overly filled and the serum leptin concentrations are high, the individual does not exhibit a normal satiety response and thus keeps eating even though they have consumed enough calories; (2) the increased leptin leads to increased activation of $\mathrm{T}$ lymphocytes which release proinflammatory molecules such as IL-2 and TNF- $\alpha$, thus contributing to the chronic low-grade inflammation, and (3) leptin 
receptors on adipose tissue protect the tissue from excess fat accumulation by stimulating fat stores to oxidize and release their fat stores into the blood however, during periods of excess energy intake, leptin receptors on adipose tissue decline and the adipose tissue develops a resistance to leptin, much like the hypothalamus.

- Once the abdominal fat stores are sufficiently hypertrophied, an automatic state of hyperlipolysis begins. The excess FFA are transported to the liver where they are converted to glucose despite the opposing mechanism of insulin. Thus, hepatic insulin resistance is developed. Once the adipose tissue is hypertrophied, the body blood supply cannot keep up with the expansion and the cells become hypoxiated. In an effort to keep the cells alive, the mitochondria of the adipocytes are placed under a lot of oxidative stress due to the increased catabolism.

- With the increase in the adhesion molecules, increased numbers of bone marrow-derived monocytes are recruited into the adipose tissue. These monocytes develop into macrophages which release larger amounts of the IL- 6 and TNF- $\alpha$ seen in obese individuals, thus contributing to the inflammation.

- The increased levels of TNF- $\alpha$ set the stage for insulin resistance and type 2 diabetes. This is achieved in a three-step process as follows: (1) increased TNF- $\alpha$ levels suppress adiponectin production, and the suppression of adiponectin leads to a reduction in the release of anti-inflammatory cytokines such as IL-1 and IL-10 - adiponectin is thus unable to inhibit the NF- $\kappa \mathrm{B}$ pathway that prevents insulin signaling; (2) TNF- $\alpha$ prevents insulin signaling by phosphorylating the insulin receptor with serine via the NF- $\kappa B$ pathway activation, and (3) TNF- $\alpha$ upregulates adhesion molecules on endothelial cells - thus, more monocytes are recruited into the adipose tissue, further adding to the inflammation and TNF- $\alpha$ levels.

- Concurrently, serum concentration of FFA increases, resulting in the following: (1) FFA inhibits insulin biosynthesis and $\beta$-cell mitogenesis; (2) FFA accumulates as ceramides in skeletal muscle - ceramides in skeletal muscle inhibit insulin signaling, thus leading to impaired glucose uptake and contributing to the hyperglycemia, and (3) FFA accumulation in the liver enhances the production of very low LDL, leading to hypertriglyceridemia gluconeogenesis and thus contributing to the hyperglycemia seen in diabetic patients.

Obesity and Antioxidants

\section{Reactive Oxygen Species}

A molecule with unpaired electrons in its outermost orbit is a free radical. When an oxygen atom is involved, it is called an oxygen free radical $[14,15]$. Reactive oxygen species may be free radicals or other oxidizing substances such as peroxides. Typically, the body oxidizes substances such as glucose to produce energy needed for living. During this process oxygen molecules are reduced in a stepwise manner to yield very reactive intermediates such as superoxide, hydrogen peroxide and hydroxyl radical [16-18]. These species occur in very low concentrations (nanomolar to micromolar). For defense purposes, cells such as macrophages may also produce large amounts of superoxide and peroxynitrite. The body uses natural antioxidants such as vitamin C (ascorbate), vitamin E, glutathione, and various enzymes to quench the reactive oxygen species. If not controlled, the excess accumulation of reactive oxygen may alter the nature of lipids and proteins and ultimately cause cellular dysfunction [16-18]. Prolonged damage due to accumulated reactive oxygen species in different tissues may cause irreversible damage. There is a recent shift in the paradigms in understanding the mechanisms of damaging actions of the reactive oxygen species since kinetic constraints indicate that in vivo scavenging of radicals is ineffective in antioxidant defense $[19,20]$. One of the mechanisms for the action of nutritional antioxidants may be oxidative activation of the $\mathrm{Nrf}$ 2 signaling pathway, leading to a mechanism termed 'para-hormesis' [19] or, with age, the redox of plasma GSH/ GSSG is not equilibrated with the larger plasma cysteine/ cystine (Cys/CySS) pool [18]. 'The major cellular thiol/ disulfide systems, including GSH/GSSG, thioredoxin-1 $(-\mathrm{SH}(2) /-\mathrm{SS}-)$, and Cys/CySS, are not in redox equilibrium and respond differently to chemical toxicants and physiologic stimuli' [18]. It is proposed that oxidative stress may be better defined as a disruption of redox signaling and control [18]. Regardless of the mechanisms of actions, the issue remains whether or not antioxidant supplementation is effective in the management of obesity and type 2 diabetes.

\section{Antioxidant Supplementation in the Management of Obesity and Type 2 Diabetes}

\section{Rationale}

How obesity is related to an increase in inflammation which is associated with an increase in oxidative stress is shown in figure 1. An increase in leptin leads to an in- 
crease in adhesion molecules; adipocytes recruit monocytes which are then transformed into macrophages. Macrophages are capable of producing large amounts of oxidants such as peroxynitrite. An obesity-associated increase in oxidative stress has been examined by different methods - measurements of thiobarbituric reactive acid substances (TBARS), malondialdehyde formation (MDA), oxidized LDL, oxidized urinary albumin, peroxide estimated from reactive oxygen assays, and the formation of carbonyl proteins in erythrocytes. A review of the literature shows that in most studies obesity and type 2 diabetes are associated with an increase in oxidative stress [21].

The usual recommended therapy for obesity-related diabetes is weight loss by decreasing calorie intake, although increased burning calories with exercise also works. A study that examined the effect of decreased calorie consumption $(500 \mathrm{kcal} /$ day less than that required for weight maintenance) with high-carbohydrate/lowprotein or high-protein/low-carbohydrate diets [22] showed that over a 6-month period, weight loss occurred in both groups. The weight loss accompanied decreased oxidative stress (as measured by two different methods), IL-6, TNF- $\alpha$, TG, FFA, CRP, adiponectin, and leptin. However, the weight loss was greater in the group on the high-protein/low-carbohydrate diet. This accompanied the various parameters above. Thus, the oxidative stress was associated with the weight loss rather than with diet alone. Given the long-term benefits observed with metformin use in diabetes patients, a study that investigated the effects of metformin on markers of oxidative stress, antioxidant reserve and HDL-C-associated antioxidant enzymes revealed that the use of metformin was more effective in reducing oxidative stress compared to lifestyle modification alone [23].

In recent years, antioxidants have been used extensively to overcome the effects of excess reactive oxygen species in several pathologies. Some of the common antioxidants used are vitamins E and C, coenzyme Q, a-lipoic acid, lycopenes, and polyphenols. Vitamin $\mathrm{E}$ is most commonly found in the form of a-tocopherol and is most abundant in dark chocolate, nuts and berries, and also as a supplement. Vitamin E requires vitamin C or coenzyme $\mathrm{Q}$ to regenerate after being used up in the free radical reaction, so studies often look at the effects of vitamin $\mathrm{C}$ and E together. Vitamin $C$ is a powerful antioxidant on its own and is most abundantly found in many fruits, broccoli and bell peppers, and also as a supplement. $\alpha$-Lipoic acid is a powerful antioxidant as it can recycle both vitamins $\mathrm{E}$ and $\mathrm{C}$, repair oxidized proteins and directly scavenge reactive oxygen species [24]. Polyphenols are a class of antioxidants which are widely available in common foods, and dietary consumption can be quite high. There are several types of polyphenols - catechins, anthocyanins, procyanidins, resveratrol, and curcumin.

Given that obesity and type 2 diabetes are associated with increased oxidative stress, the question is whether antioxidant supplementation has a place in their therapy. To answer this question, PubMed database was used to search for all the literature. The most recent searches were conducted in May and June of 2014. The initial search was conducted for the presence of the following term combinations in the abstracts: 'obesity or weight loss or type 2 diabetes' and 'antioxidants' and 'treatment or therapy or prevention' and 'human or clinical trial'. Searches were then conducted with the term antioxidants replaced by the terms for individual antioxidants. The pertinent literature cited in the relevant publications was also examined. Where needed, searches were also conducted for the various molecular markers of obesity.

The antioxidants considered here are in the following two major categories: (1) vitamins and cofactors and (2) polyphenols and carotenoids.

\section{Antioxidant Vitamins and Cofactors}

\section{Vitamins $\mathrm{C}$ and $\mathrm{E}$}

Vitamins $\mathrm{C}$ and $\mathrm{E}$ are cofactors in many enzymatic reactions and also antioxidants. Vitamin $\mathrm{E}$ acts as a peroxyl scavenger and thus prevents oxidation of membrane lipids [25]. Vitamin $\mathrm{E}$ is present in small amounts and the recycling of its oxidized form to the reduced form is coupled with vitamin $\mathrm{C}$ which is present in the body at a much higher concentration. However, the human body cannot synthesize vitamin $\mathrm{C}$ and normally obtains it from foodstuff. Several studies have examined the effects of these vitamins in type 2 diabetes [26-30].

To examine whether there is a relationship between vitamin $\mathrm{C}$ levels and the risk of type 2 diabetes, a long-term study using food frequency questionnaires was conducted, and a significant inverse relationship between vitamin C levels and the incidence of diabetes was observed [26]. The difficulty in interpreting this observation is that the vitamin C levels were directly related to the consumption of fruits and vegetables and could also be attributed to education, physical activity and other lifestyle-related issues. In a random double-blind study, type 2 diabetes patients (aged 30-60 years, BMI >25) were given a placebo, vitamin $\mathrm{C}$, or vitamin $\mathrm{E}$ (or vitamins $\mathrm{C}$ plus $\mathrm{E}$ ) for a period of 3 months. In all the supplement groups, the level of hypertension decreased. The initial values of vitamin $\mathrm{C}, \mathrm{E}$ and $\mathrm{E}$ plus $\mathrm{C}$ compared to the placebo group showed significant 
differences in the concentration of FBG and glycated hemoglobin $\left(\mathrm{HbA}_{1 \mathrm{c}}\right)$, superoxide dismutase and glutathione peroxides enzymes levels [27]. In a study on menopausal women with diabetes, the effects of hormone replacement therapy with and without vitamin $\mathrm{C}$ and $\mathrm{E}$ supplements were examined. The therapy decreased lipid peroxidation and showed significant differences in glutathione peroxidase, reduced glutathione, $\beta$-carotene, catalase, plasma glucose, LDL, and TG [28]. Supplementation with vitamins $C$ plus $E$ further decreased lipid peroxidation. In a study on long-term intake of antioxidants on male smokers, the antioxidant supplementation was ineffective in preventing diabetes-related complications [29]. One study even claimed that vitamin $\mathrm{C}$ and $\mathrm{E}$ supplementation may cancel the beneficial effects of exercise, but this study was not conducted on diabetic or obese subjects [30].

To summarize, there may be clear benefits of longterm consumption of vitamin $\mathrm{C}$ through diets containing fruits and vegetables, but the effects of vitamin $\mathrm{C}$ and $\mathrm{E}$ supplementation are marginal.

\section{Zinc}

Zinc is a cofactor for several antioxidant enzymes such as $\mathrm{Cu} \mathrm{Zn}$ superoxide dismutase [31]. Zinc supplements have been tested for effects on oxidative stress and type 2 diabetes.

A questionnaire-based observational study of Australian women (8,921 subjects, aged 50-55 years at end of study) over a 10-year period concluded that higher dietary zinc intake decreased the risk of developing type 2 diabetes [32]. There are some potential issues with the method used, but the sample size is large.

A study on Iranian patients already taking metformin showed that supplementation with zinc and melatonin decreased the levels of $\mathrm{HbA}_{1 \mathrm{c}}$ after only 30 days and further significantly improved FBG after 90 days [33]. Zinc supplementation has also been shown to improve glycemic control and lipid profile in patients with higher FBG or higher postprandial glucose levels [34]. In a Tunisian study, zinc gluconate and/or chromium for 6 months had no effect on $\mathrm{HbA}_{1 \mathrm{c}}$ or glucose homeostasis, although membrane oxidation decreased [35]. It was noted that the activity of the $\mathrm{Cu} \mathrm{Zn}$ superoxide dismutase did not show a change. A double-blind crossover study on male type 2 diabetic patients taking glibenclamide showed a decrease in cholesterol and an increase in HDL but no changes in FBG or $\mathrm{HbA}_{1 \mathrm{c}}$ [36]. Some studies found no benefits of zinc supplementation in diabetic patients $[37,38]$.

In 2012, a meta-analysis was conducted of the effects of zinc supplementation on diabetes [39]. The studies ana- lyzed were very different in terms of the amounts supplemented and whether or not there was something else also supplemented at the same time. The conclusion was that zinc did have a beneficial effect on FBG, postprandial plasma glucose and lipid profiles. The benefit may be even greater in patients who are more poorly controlled by medication or who are more overweight and hence might have higher oxidative stress levels and lower zinc levels.

\section{Lipoic Acid}

Lipoic acid is made in small amounts in the human body. It is a cofactor for enzymes involved in two oxoacid dehydrogenase enzymes such as the pyruvate dehydrogenase complex, the 2-oxoglutarate dehydrogenase complex, the branched-chain oxoacid dehydrogenase complex, and the acetoin dehydrogenase complex [40, 41]. Of particular importance is the pyruvate dehydrogenase complex which is involved directly in energy metabolism. It is also an antioxidant which is found in certain foods, including red meat, spinach, broccoli, potatoes, yams, carrots, beets, and yeast. However, the lipoic acid content is too low in the foods and, also, it is not readily bioavailable due to its conjugation to lysine [42]. It has been clinically tested as a food supplement for its potential benefits in obesity and diabetes.

A study examined the effect of $800 \mathrm{mg} /$ day lipoic acid supplementation in 445 men and 682 women aged 18-60 years and obese or preobese [43]. The study considered dietary and other parameters but had no placebo groups. It found significant benefits of the supplementation to anthropometric parameters of obesity such as weight and $\mathrm{BMI}$ and the inflammation markers such as CRP, TNF- $\alpha$ and IL-6. In a random trial in Iran, capsules containing $300 \mathrm{mg}$ lipoic acid or placebo were given daily for 8 weeks to type 2 diabetics [44]. There was a significant decrease in FBG, postprandial glucose and insulin resistance with lipoic acid. Another study with a cohort of 12 patients on the effects of oral lipoic acid supplementation showed that it increased the insulin sensitivity in diabetic patients [45]. A study over a 12 -week period on obese patients concluded that $\alpha$-lipoic acid ingestion $(1,000 \mathrm{mg} /$ day $)$ may increase the atherogenicity of LDL when ingested without exercise and may thus contribute to the pathogenesis of atherosclerosis and the development of cardiovascular disease. However, when combined with exercise, this atherogenic effect was abolished and the supplementation was beneficial [46]. In a double-blind placebo-controlled 20-week trial, 360 obese individuals were randomized for treatment with oral $\alpha$-lipoic acid 1,200 or 1,800 $\mathrm{mg} /$ day or a placebo [47]. The treatment led to a signifi- 
cantly greater weight loss $(2.1 \%)$ than in the placebo group.

Intravenous administration of $600 \mathrm{mg}$ or a placebo once daily for 2 weeks was conducted in a double-blind control study on 22 obese subjects [48]. The treatment improved insulin sensitivity and had marginal but statistically significant beneficial effects on FFA, TG, total cholesterol, plasma oxidative products, adiponectin, and the inflammation markers TNF- $\alpha$ and IL-6.

In contrast, the results were different in other studies. One study found that lipoic acid decreased oxidative stress and increased HDL but had no significant effect on total cholesterol, TG or CRP [49]. Another study concluded that $\alpha$-lipoic acid administered orally did not protect against lipid-induced insulin resistance in overweight and obese subjects [50].

Overall, lipoic acid is needed for the body and may be synthesized in sufficient amounts. Dietary lipoic acid is not readily bioavailable. However, lipoic acid supplementation may be beneficial to some degree in obesity and diabetes. It has also been recommended for other diseases such as cancer.

\section{Carnitine}

L-Carnitine is involved in the conversion of FFA into usable energy. It transports FFA into the mitochondrial matrix so that they can be metabolized for the conversion of energy via the citric acid cycle [51]. It is found in red meat, fish, dairy products, soy, nuts, and seeds. It may lower oxidative stress and has been studied extensively for benefits in type 2 diabetes.

A random double-blind study compared the effects of orlistat with and without carnitine in 258 patients with uncontrolled diabetes over 1 year [52]. Compared to orlistat alone, orlistat plus L-carnitine gave a better improvement in body weight, glycemic control, lipid profile, and inflammatory markers. However, the benefits of carnitine were marginal, and the patients were also concurrently taking different medications for the control of diabetes. Another study from the same group compared the effects of sibutramine with and without carnitine [53]. Carnitine caused a larger decrease in FBG and $\mathrm{HbA}_{1 \mathrm{c}}$ and also had small effects on weight loss and BMI. Two other studies compared the effects of simvastatin with and without L-carnitine $[54,55]$. In both studies, carnitine had a significant benefit in lowering FBG and TG and improving cholesterol and LDL-related metabolism.

A meta-analysis of four studies [56] on the effects of oral carnitine supplementation revealed that oral carnitine lowered FBG, total cholesterol, apolipoprotein-B100, and apolipoprotein-A1, but changes in $\mathrm{TG}, \mathrm{HbA}_{1 \mathrm{c}}$ or lipoprotein A were not significant. It is not clear why the meta-analysis was limited to only four studies.

All the studies were not listed in this area, but in general most of them showed carnitine supplementation to be beneficial for diabetes, even though a number of the studies were conducted poorly and the benefits were only marginal in some of them.

\section{Polyunsaturated Fatty Acids}

Omega-3 polyunsaturated fatty acids (LCn-3PUFA) have been shown to contribute to human health [57]. Foods rich in them are fish, flax seeds and walnuts, and even eggs rich in omega-3 rich are available. Obesity has been linked with low levels of LCn-3PUFA [58].

In a randomized double-blind study, a very low-energy diet led to a loss of fat and weight. LCn-3PUFA accelerated the process [59]. A crossover diet study on 17 obese and nonobese subjects for a 5 -week period showed that LCn-3PUFA did not affect body weight, BMI, cholesterol (total, HDL or LDL), insulin sensitivity, TG, nonesterified fatty acids, glycerol, lactate, glucose, or $\mathrm{HbA}_{1 \mathrm{c}}[60]$.

\section{Coenzyme Q10}

Coenzyme Q10 is involved in the mitochondrial electron transport chain which is essential for energy production [61]. Organs rich in this substance are the heart, liver and muscle. It is present in meat (beef, pork and chicken) and in soybeans, olive oil, grape seeds, nuts, and some fruits and vegetables. Due to its relationship to energy metabolism, it has been tested for obesity and diabetes.

A double-blind placebo-controlled study over 12 weeks showed that coenzyme Q10 supplementation in obese subjects did not affect inflammatory markers, arterial stiffness or fatigue [62]. In other studies coenzyme Q10 supplementation may have improved endothelial function and produced antihypertensive effects, but there were no reproducible effects on body weight, fat mass or glycemia $[63,64]$. A literature review concluded that antiinflammatory effects of coenzyme Q10 have been described in vitro, but supplementation seems to have only an antihypertensive effect, with no benefits for body weight, fat mass or glycemia [65].

\section{Polyphenols and Carotenoids}

\section{Procyanidins and Cinnamon}

Procyanidin-rich foods are cinnamon, apple, cranberries, avocado, red beans, almonds, and peanuts, but cin- 
namon has been tested most extensively, possibly because it contains different varieties of procyanidins [66]. Cinnamon is used as a spice to add flavor to dishes but has also been recommended in Ayurvedic and Chinese medicine, possibly because of its tannin content. Cinnamon cassia (Cinnamomum cassia) is rich in cinnamaldehyde and has been studied extensively in improving the health of diabetics [67-73]. C. cassia contains coumarin which may be toxic in large doses, but the extracts from this plant are not as rich in coumarin and have been used in many studies.

Several studies have shown C. cassia to be beneficial for diabetes. A blind study using 3 different amounts of cinnamon (1, 3 or $6 \mathrm{~g}$ ) over the course of 40 days with a 20 day follow-up showed that cinnamon significantly lowered FBG and that the effect persisted after a 20 -day washout period. Cinnamon also decreased fasting TG and cholesterol levels [67]. In another random blind study the cinnamon extract of $C$. cassia decreased FBG after 4 months of treatment, but there was no effect on levels of $\mathrm{HDL}, \mathrm{LDL}, \mathrm{HbA}_{1 c}$, or TG [68]. In Chinese diabetic patients simultaneously taking gliclazide, cinnamon extract caused a decrease in FBG and $\mathrm{HbA}_{1 \mathrm{c}}$ but not in cholesterol or TG [69]. Yet another study showed that cinnamon extract lowered FBG, oxidative stress and fat mass and increased the lean body mass over a 12 -week period but not over a 6-week period [70]. One study used cinnamon extract-containing capsules over the counter. The capsules were taken daily, but the study was not blind or controlled for diet and exercise. There was a lowering of $\mathrm{HbA}_{1 \mathrm{c}}$ by $0.83 \%$ among the treatment group [71]. In contrast, several studies showed no effects of cinnamon. In a random study with postmenopausal women it did not alter blood lipid profile, oral glucose tolerance or FBG [72]. However, the subjects in this group were also on a variety of treatments (excluding insulin) to control their diabetes. Another study also found no effect of cinnamon on BMI, FBG, cholesterol, TG, or insulin levels [73].

In our opinion, the studies using cinnamon are at best poorly conducted and hence the results may vary. First, some of the ingredients in cinnamon are coumarin, cinnamic acid, cinnamaldehyde, cinnamyl alcohol, and eugenol. There was a 5- to 10-fold variation in the concentration of these ingredients in different samples [74]. This was because the quality of cinnamon depends on which branches were used and how they were processed. The studies did not provide the contents of these ingredients in the materials used. Most of the work discussed here used C. cassia (Chinese cinnamon). Cinnamomum zeylanicum (Sri Lankan cinnamon) has been tested extensive- ly in animals and shown to be beneficial in diabetes, but the human studies mostly used C. cassia [75]. The effectiveness of cinnamon or cinnamon extracts may depend on how well controlled the diabetes was at the beginning of the study. Each study used different parameters and different time periods and most were small studies, which makes comparisons complicated.

\section{Catechins and Green Tea}

The beneficial health effects of tea and green tea have been celebrated in many cultures where tea is the most commonly consumed beverage. However, the word tea is also used as generic term for herbal drinks made in hot water and, therefore, it is clarified that this section focuses on tea made from the leaves of Camellia sinensis. Tea may be processed and brewed or used as dried green leaves - hence the term green tea which is the focus here. Green tea is rich in catechins and caffeine but also contains vitamins $\mathrm{B}, \mathrm{C}$ and $\mathrm{E}$, carotene and theanine [76].

In a randomized double-blind study conducted over a 12 -week period on a selected overweight $(\mathrm{BMI}>25)$ Thai population, the food provided 2,000 kcal/day, and energy expenditure and physical activity were measured [77]. The cohort consuming the green tea supplement lost significantly more weight than the placebo group $(\mathrm{p}<0.05)$. The weight loss peaked at 8 weeks, as did the energy expended. The tea was hypothesized to increase the energy expended through thermogenesis and fat oxidation. This is consistent with the weight loss since the actual activity level or the caloric intake did not change. An open study conducted in France using 68 overweight to moderately obese patients found a weight loss of $4.6 \%$ over 12 weeks taking a green tea supplement (AR25, 375 mg 4 times/ day) containing caffeine [78]. This was a completely open study with no control group and it did not mention diet or activity levels during the course of the study. Interestingly, another open study did not have a significant effect of decaffeinated green tea or green tea supplements over 8 weeks on biomarkers of inflammation in obese subjects [79]. The fact that this study used decaffeinated green tea/ supplement might have had an effect on the results, suggesting that the observed benefits were possibly due to a synergy between catechins and caffeine. There was no diet control in this study and no recording of normal caffeine intake. There may be a point at which too much caffeine ceases to help in weight loss as the body develops a tolerance to caffeine (proposed to be more than $\sim 300 \mathrm{mg}$ / day) $[80,81]$. Some populations may be consuming, in a normal day, from 0.5 to 1 liter of coffee in North America or in more concentrated coffee drinks in Europe, as in 
Turkish coffee or the Arabic kahva. Even several soft drinks may contain sufficient caffeine.

In a study of whether or not a combination of caffeine and catechins could influence weight maintenance after a period of weight loss, it was found that in those who habitually consumed a lower amount of caffeine $(<300 \mathrm{mg} /$ day), weight was maintained, and even further weight was lost compared with habitual high caffeine consumers or those taking placebo, all of whom gained weight over the same 3-month period [80].

The degree to which green tea is effective may change from population to population and over time as some of the effect is due to caffeine - the Thai population consumes less caffeine than the North American or European population - and a tolerance to caffeine can develop. Also, it is noted that the exact chemical composition of green tea may depend on the cultivars, the region where it is grown, seasonality of harvest, which leaves are harvested, and how the green tea is processed and prepared.

It is pointed out that the customs in tea preparations vary and some of them may be beneficial for diabetes. For example, Kashmiri tea also contains cinnamon and a trace of saffron, which may be beneficial for diabetes [82]. The typical Indian masala tea does contain the antioxidant containing beneficial spices but is typically made with sugar and milk, which may increase the calorie and fat consumption.

\section{Chlorogenic Acid Green Coffee Extract}

Chlorogenic acid is an antioxidant found in plums (prunes), peaches, potatoes, and date palm, as well as in green coffee beans. Green coffee is trendy and publicized on the Internet for its weight loss potential. However, there are very few reliable studies to support this claim. There are several small short-term ill-controlled studies $[83,84]$. A small double-blind crossover trial with 16 preobese participants showed that green coffee extract supplementation resulted in weight loss over a 22 -week period [83]. A meta-analysis in 2011 concluded the results with green coffee to be promising but questions the quality of these studies [84]. Larger-scale well-conducted trials on diabetic patients were not found.

Our opinion is that green coffee may be marginally useful due to its chlorogenic acid content. However, this antioxidant is also available in fruits such as prunes and dates which also contain ferulic acid - another antioxidant which has proved useful in diabetes studies in animals but has not yet been tested in humans $[85,86]$. Would the use of these fruits in a regular diet not be more beneficial than the green coffee extract supplements?

\section{Resveratrol}

Resveratrol is a polyphenolic compound found in the skin of red grapes, in fruits such as pomegranates, in berries such as acai and Ziziphus, and in the roots of Japanese knotweed (Polygonum cuspidatum). Red wine is also rich in this antioxidant. It has been shown to be of benefit in cardiovascular function and investigated for potential benefits in diabetes [87].

In a double-blind study using 19 type 2 diabetic men who were already on oral glucose-lowering drugs, the effect of $5 \mathrm{mg}$ capsules ( $2 \times$ daily for 4 weeks) containing resveratrol or placebo was studied [88]. Resveratrol significantly decreased oxidative stress and insulin resistance. In an open-label randomized control study the effect of 250 $\mathrm{mg}$ of resveratrol on type 2 diabetic subjects on oral hypoglycemic treatment with metformin and/or glibenclamide was compared with a placebo group [89]. After 3 months of treatment, the resveratrol group showed a marginal but statistically significant improvement in mean $\operatorname{HbA}_{1 \mathrm{c}}(\mathrm{p}=$ $0.02)$, systolic blood pressure $(p=0.0002)$ and total cholesterol $(p<0.004)$ but not in body weight $(p=0.83)$, FBG $(\mathrm{p}=0.29)$, HDL $(\mathrm{p}=0.42)$, or LDL $(\mathrm{p}=0.05)$.

The benefits of this antioxidant have been even fewer in other studies [90-92]. A study using up to $2 \mathrm{~g}$ of resveratrol daily for 4 weeks showed a modest decrease in postprandial glucose levels but not in FBG and any other measures [90]. In another study, the effects of $500 \mathrm{mg}$ resveratrol for 4 weeks in healthy obese men compared to those on placebo showed that endogenous glucose production and the turnover and oxidation rates of glucose remained unchanged [91]. Resveratrol supplementation also had no effect on blood pressure, resting energy expenditure, oxidation rates of lipids, ectopic or visceral fat content, or inflammatory and metabolic biomarkers. The study by Yoshino et al. [92] on nonobese postmenopausal women using $75 \mathrm{mg}$ of this supplement for 12 weeks did not reveal any benefits for body composition, resting metabolic rate, plasma lipids, or inflammatory markers.

Our opinion is that this antioxidant may be beneficial for health in general, but even as an adjunct therapy for type 2 diabetes its usefulness remains to be established.

\section{Lycopene}

Lycopene-rich foods are common and include guava, papaya, watermelon, and solanaceous plants such as tomato, eggplant and potato. Lycopene is a carotenoid and is an antioxidant which has been studied for its anti-inflammatory properties, most often in conjunction with cancer or cardiovascular disease but not as extensively or systematically for obesity or diabetes [93]. 
There are several small studies on the therapeutic effects of lycopene on diabetes [94-96]. They did not show any benefits in anthropomorphic data, FBG, $\mathrm{HbA}_{1 c}$, or other markers for impaired glucose tolerance, but some studies showed that it does not have any added value apart from marginal effects on oxidative stress status and inflammation markers IL-6, TNF- $\alpha$ or CRP.

\section{Synopsis, Critical Appraisal and Recommendations}

\section{Synopsis}

The WHO defines obesity as a condition of abnormal or excessive fat accumulation in adipose tissue, such that health may be impaired. Clinically, in adults obesity is defined as having a BMI $>30$. However, this definition is inadequate since it does not include the abnormal distribution of fat which is the main risk factor for obesity and its comorbidities. The adipocytes in the visceral fat can recruit monocytes which are transformed into macrophages. The adipocytes and macrophages can produce molecules which lead to the metabolic syndrome and release of FFA, terminating in type 2 diabetes. Obesity and type 2 diabetes are associated with an increase in oxidative stress. Therefore, it would be logical to consider antioxidant supplementation in potential therapy for obesity and diabetes. Here we considered the following types of antioxidants: vitamins and cofactors, polyphenols and carotenoids. Table 1 contains a summary of the key observations.

Briefly, there may be some benefits of long-term consumption of vitamin $\mathrm{C}$ through diets containing fruits and vegetables, but the effects of vitamin $C$ and $E$ supplementation are marginal. Zinc supplements may have benefits which include lowering FBG, postprandial plasma glucose and lipid profiles - and may be even greater when control with medication is poor. Lipoic acid supplementation may be beneficial to some degree in obesity and diabetes. Carnitine is beneficial for diabetes in lowering FBG, total cholesterol, apolipoprotein-B100, and apolipoprotein-A1 but has no effects on TG, $\mathrm{HbA}_{1 c}$ or lipoprotein A. For diabetics, the evidence for the benefits of LCn-3PUFA and coenzyme Q10 is feeble, although the latter seems to have an antihypertensive effect.

There is reasonable evidence for benefits of cinnamon in lowering $\mathrm{FBG}, \mathrm{HbA}_{1 \mathrm{c}}$, oxidative stress, and possibly fat mass. However, the effectiveness of cinnamon or cinnamon extracts may depend on how well controlled the diabetes is at the beginning of the study. Green tea (not decaffeinated) may increase thermogenesis and fat oxida- tion, leading to small weight loss. Chlorogenic acid in green coffee has, if any, only a small potential for weight loss. Resveratrol reduces oxidative stress and had marginal benefits for diabetes in some studies but not in others. Lycopene may have marginal benefits in decreasing oxidative stress status, IL-6, TNF- $\alpha$, or CRP but has no effects on weight, $\mathrm{FBG}, \mathrm{HbA}_{1 \mathrm{c}}$, or impairment of glucose tolerance.

Thus, supplementation with zinc, lipoic acid, carnitine, cinnamon, green tea, and possibly vitamin $\mathrm{C}$ plus $\mathrm{E}$ had marginal benefits for diabetes. However, the evidence for such benefits from LCn-3PUFA, coenzyme Q10, green coffee, resveratrol, and lycopene was less than convincing. Recall that only the potential benefits for obesity and diabetes were examined here. However, the various other antioxidants could also have other health benefits which were not considered.

\section{Critical Appraisal}

There are several issues in considering the benefits of antioxidants in obesity and type 2 diabetes. The first is the confusion over the definition of obesity. Typically, this definition is based on BMI, which is inadequate, as are the other anthropomorphic measurements. The concern becomes significantly greater when FBG levels become very high and the risk of insufficiency of secretion and functioning of insulin is realized - then the disease is diagnosed as diabetes. However, there is a realm of metabolic changes that occur in between, and these may be gradual but chronic and cause low-grade inflammation. Therefore, the researchers investigating the benefits of antioxidants face the dilemma concerning at which stage can subjects be recruited into the studies and what parameters should be measured. Subjects who are diabetic or near diabetic may be easier to recruit and have greater compliance than those in early stages of obesity where the metabolic syndrome may have begun to set in. Consequently, there is considerable heterogeneity in the literature - in the types of subjects used and the measurements made. Furthermore, subjects in the advanced stages may already be on different medications, thereby producing the complication of interactions between various treatments and the antioxidants. Another issue is that money is not as plentiful in this field as it is for drug company research. The net result of all these problems is that most studies are small both in the number of subjects and duration. They also often lack appropriate controls. Many are epidemiological studies or meta-analyses, using the self-reporting of antioxidant intake, which is notoriously prone to error. Also, for intervention studies when natural 
Table 1. Summary of benefits of antioxidants in obesity and type 2 diabetes

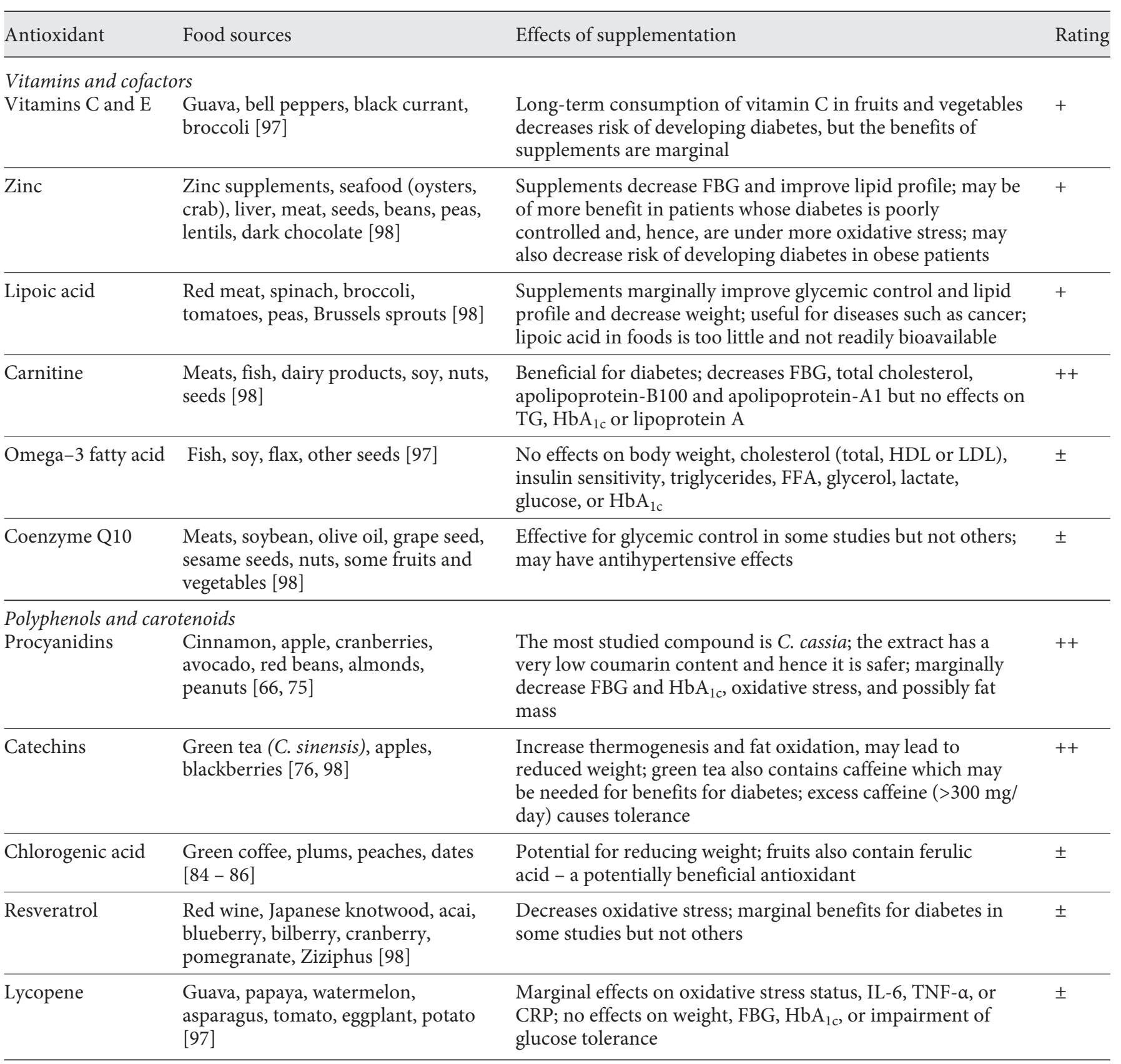

Rating is based on the strength of evidence for the effectiveness of the antioxidants for diabetes/obesity management.

products are used, their key antioxidant determinants are not reported. The product quality, stability and absorption of the same antioxidant can vary considerably between the various manufacturers. The source of the intervention is frequently not reported, and this can have a significant impact on whether or not a particular antioxidant will prove to exert a clinical benefit. Also, the bias due to source of funding and other conflicts of interest cannot be ignored. Hence, it was difficult for us to come up with laser-sharp conclusions for each substance. 


\section{Recommendations}

In most cultures, the availability of plentiful food was a sign of success and satisfaction. However, in today's society this should no longer be true due to a decrease in the amount of physical activity and an increase in high-calorie foods. Whether it be preobese, obese or type 2 diabetic, the therapy has to begin with an increase in exercise and a decrease in calorie consumption. Having made this statement, it is also clear that antioxidants do have a role in slowing the low-grade inflammation associated with these diseases. The ideal would be increasing foods rich in antioxidants as part of a lifestyle from an early age (table 1). For those who are in more advanced stages of the disease, supplementation with a combination of antioxidants may also be beneficial. However, ideally it would be the change in lifestyle that leads to the consumption of a balanced diet low in calories and rich in antioxidants. The literature does not suggest antioxidant supplementation as a cure-all for obesity or for type 2 diabetes.

\section{Acknowledgment}

This work was supported by internal funds from McMaster University. No other funding was received.

\section{Disclosure Statement}

The authors have no conflicts of interest to declare.

\section{References}

1 Behbehani K: Kuwait National Programme for Healthy Living: first 5-year plan (20132017). Med Princ Pract 2014;23(suppl 1):3242.

2 Hotamisligil GS: Inflammation and metabolic disorders. Nature 2006;444:860-867.

13 World Health Organization. Obesity: preventing and managing the global epidemic. http://www.who.int/nutrition/publications/ obesity/WHO_TRS_894/en/.2000.

4 Bruckert E: Abdominal obesity: a health threat (in French). Presse Med 2008;37:14071414.

5 Despres JP, Lemieux I: Abdominal obesity and metabolic syndrome. Nature 2006;444: 881-887.

6 Schelbert KB: Comorbidities of obesity. Prim Care 2009;36:271-285.

-7 Al Lawati JA, Barakat NM, Al Lawati AM, et al: Optimal cut-points for body mass index, waist circumference and waist-to-hip ratio using the Framingham coronary heart disease risk score in an Arab population of the Middle East. Diab Vasc Dis Res 2008;5:304-309.

8 Brennan DD, Whelan PF, Robinson K, et al: Rapid automated measurement of body fat distribution from whole-body MRI. AJR Am J Roentgenol 2005; 185:418-423.

$\checkmark 9$ Hofker M, Wijmenga C: A supersized list of 21 obesity genes. Nat Genet 2009;41:139-140.

$>10$ Wood IS, Trayhurn P: Glucose transporters (GLUT and SGLT): expanded families of sugar transport proteins. Br J Nutr 2003;89:3-9.

-11 Foley K, Boguslavsky S, Klip A: Endocytosis, recycling, and regulated exocytosis of glucose transporter 4. Biochemistry 2011;50:30483061.

12 Fantuzzi G: Adipose tissue, adipokines, and inflammation. J Allergy Clin Immunol 2005; 115:911-919.
13 Wisse BE: The inflammatory syndrome: the role of adipose tissue cytokines in metabolic disorders linked to obesity. J Am Soc Nephrol 2004;15:2792-2800.

14 Walia M, Kwan CY, Grover AK: Effects of free radicals on coronary artery. Med Princ Pract 2003;12:1-9.

15 Fridovich I: Oxygen: how do we stand it? Med Princ Pract 2013;22:131-137.

16 Giacco F, Brownlee M: Oxidative stress and diabetic complications. Circ Res 2010;107: 1058-1070.

$\checkmark 17$ Rudich A, Kanety H, Bashan N: Adipose stress-sensing kinases: linking obesity to malfunction. Trends Endocrinol Metab 2007;18: 291-299.

18 Jones DP: Redefining oxidative stress. Antioxid Redox Signal 2006;8:1865-1879.

Forman HJ, Davies KJ, Ursini F: How do nutritional antioxidants really work: nucleophilic tone and para-hormesis versus free radical scavenging in vivo. Free Radic Biol Med 2014; 66:24-35.

20 Liochev SI: Free radicals: how do we stand them? Anaerobic and aerobic free radical (chain) reactions involved in the use of fluorogenic probes and in biological systems. Med Princ Pract 2014;23:195-203.

21 Vincent HK, Innes KE, Vincent KR: Oxidative stress and potential interventions to reduce oxidative stress in overweight and obesity. Diabetes Obes Metab 2007;9:813-839.

22 Kitabchi AE, McDaniel KA, Wan JY, et al: Effects of high-protein versus high-carbohydrate diets on markers of beta-cell function, oxidative stress, lipid peroxidation, proinflammatory cytokines, and adipokines in obese, premenopausal women without diabetes: a randomized controlled trial. Diabetes Care 2013;36:1919-1925.
23 Esteghamati A, Eskandari D, Mirmiranpour $\mathrm{H}$, et al: Effects of metformin on markers of oxidative stress and antioxidant reserve in patients with newly diagnosed type 2 diabetes: a randomized clinical trial. Clin Nutr 2013;32: 179-185.

24 Golbidi S, Badran M, Laher I: Diabetes and alpha lipoic acid. Front Pharmacol 2011;2:69.

25 Niki E: Role of vitamin E as a lipid-soluble peroxyl radical scavenger: in vitro and in vivo evidence. Free Radic Biol Med 2014;66:3-12.

26 Harding AH, Wareham NJ, Bingham SA, et al: Plasma vitamin C level, fruit and vegetable consumption, and the risk of new-onset type 2 diabetes mellitus: the European prospective investigation of cancer - Norfolk prospective study. Arch Intern Med 2008;168:1493-1499.

27 Rafighi Z, Shiva A, Arab S, Mohd YR: Association of dietary vitamin $\mathrm{C}$ and $\mathrm{E}$ intake and antioxidant enzymes in type 2 diabetes mellitus patients. Glob J Health Sci 2013;5:183-187.

28 Naziroglu M, Simsek M, Simsek H, et al: The effects of hormone replacement therapy combined with vitamins $\mathrm{C}$ and $\mathrm{E}$ on antioxidants levels and lipid profiles in postmenopausal women with type 2 diabetes. Clin Chim Acta 2004;344:63-71.

29 Kataja-Tuomola MK, Kontto JP, Mannisto S, et al: Intake of antioxidants and risk of type 2 diabetes in a cohort of male smokers. Eur J Clin Nutr 2011;65:590-597.

30 Ristow M, Zarse K, Oberbach A, et al: Antioxidants prevent health-promoting effects of physical exercise in humans. Proc Natl Acad Sci USA 2009;106:8665-8670.

31 Rotilio G, Calabrese L, Bossa F, et al: Properties of the apoprotein and role of copper and zinc in protein conformation and enzyme activity of bovine superoxide dismutase. Biochemistry 1972;11:2182-2187. 
32 Vashum KP, McEvoy M, Shi Z, et al: Is dietary zinc protective for type 2 diabetes? Results from the Australian longitudinal study on women's health. BMC Endocr Disord 2013; 13:40.

33 Hussain SA, Khadim HM, Khalaf BH, et al: Effects of melatonin and zinc on glycemic control in type 2 diabetic patients poorly controlled with metformin. Saudi Med J 2006;27: 1483-1488.

-34 Gunasekara P, Hettiarachchi M, Liyanage C, et al: Effects of zinc and multimineral vitamin supplementation on glycemic and lipid control in adult diabetes. Diabetes Metab Syndr Obes 2011;4:53-60.

35 Roussel AM, Kerkeni A, Zouari N, et al: Antioxidant effects of zinc supplementation in Tunisians with type 2 diabetes mellitus. J Am Coll Nutr 2003;22:316-321.

- 36 Partida-Hernandez G, Arreola F, Fenton B, et al: Effect of zinc replacement on lipids and lipoproteins in type 2 diabetic patients. Biomed Pharmacother 2006;60:161-168.

- 37 Seet RC, Lee CY, Lim EC, et al: Oral zinc supplementation does not improve oxidative stress or vascular function in patients with type 2 diabetes with normal zinc levels. Atherosclerosis 2011;219:231-239.

- 38 Parham M, Amini M, Aminorroaya A, et al: Effect of zinc supplementation on microalbuminuria in patients with type 2 diabetes: a double-blind, randomized, placebo-controlled, cross-over trial. Rev Diabet Stud 2008; 5:102-109.

- 39 Jayawardena R, Ranasinghe P, Galappatthy P, et al: Effects of zinc supplementation on diabetes mellitus: a systematic review and metaanalysis. Diabetol Metab Syndr 2012;4:13.

-40 Radke GA, Ono K, Ravindran S, et al: Critical role of a lipoyl cofactor of the dihydrolipoyl acetyltransferase in the binding and enhanced function of the pyruvate dehydrogenase kinase. Biochem Biophys Res Commun 1993; 190:982-991.

41 Wagenknecht T, Frank J: Localization of lipoyl-bearing domains in the alpha-ketoglutarate dehydrogenase multienzyme complex. Biochemistry 1984;23:3383-3389.

42 Biewenga GP, Haenen GR, Bast A: The pharmacology of the antioxidant lipoic acid. Gen Pharmacol 1997;29:315-331.

-43 Carbonelli MG, Di Renzo L, Bigioni M, et al: a-Lipoic acid supplementation: a tool for obesity therapy? Curr Pharm Des 2010;16:840846.

44 Ansar H, Mazloom Z, Kazemi F, et al: Effect of $\alpha$-lipoic acid on blood glucose, insulin resistance and glutathione peroxidase of type 2 diabetic patients. Saudi Med J 2011;32:584588.

-45 Kamenova P: Improvement of insulin sensitivity in patients with type 2 diabetes mellitus after oral administration of $\alpha$-lipoic acid. Hormones (Athens) 2006;5:251-258.
46 McNeilly AM, Davison GW, Murphy MH, et al: Effect of a-lipoic acid and exercise training on cardiovascular disease risk in obesity with impaired glucose tolerance. Lipids Health Dis 2011;10:217.

47 Koh EH, Lee WJ, Lee SA, et al: Effects of a-lipoic acid on body weight in obese subjects. Am J Med 2011;124:85-88.

48 Zhang Y, Han P, Wu N, et al: Amelioration of lipid abnormalities by a-lipoic acid through antioxidative and anti-inflammatory effects. Obesity (Silver Spring) 2011;19:1647-1653.

49 Gianturco V, Bellomo A, D’Ottavio E, et al: Impact of therapy with $\alpha$-lipoic acid (ALA) on the oxidative stress in the controlled NIDDM: a possible preventive way against the organ dysfunction? Arch Gerontol Geriatr 2009; 49(suppl 1):129-133.

50 Xiao C, Giacca A, Lewis GF: Short-term oral a-lipoic acid does not prevent lipid induced dysregulation of glucose homeostasis in obese and overweight nondiabetic men. Am J Physiol Endocrinol Metab 2011;301:E736-E741.

51 Normann PT, Flatmark T: Long-chain acylCoA synthetase and 'outer' carnitine longchain acyltransferase activities of intact brown adipose tissue mitochondria. Biochim Biophys Acta 1978;530:461-473.

52 Derosa G, Maffioli P, Ferrari I, et al: Comparison between orlistat plus L-carnitine and orlistat alone on inflammation parameters in obese diabetic patients. Fundam Clin Pharmacol 2011;25:642-651.

53 Derosa G, Maffioli P, Salvadeo SA, et al: Sibutramine and L-carnitine compared to sibutramine alone on insulin resistance in diabetic patients. Intern Med 2010;49:17171725.

54 Malaguarnera M, Vacante M, Motta M, et al: Effect of L-carnitine on the size of low-density lipoprotein particles in type 2 diabetes mellitus patients treated with simvastatin. Metabolism 2009;58:1618-1623.

55 Galvano F, Li VG, Malaguarnera M, et al: Effects of simvastatin and carnitine versus simvastatin on lipoprotein(a) and apoprotein(a) in type 2 diabetes mellitus. Expert Opin Pharmacother 2009;10:1875-1882.

56 Vidal-Casariego A, Burgos-Pelaez R, Martinez-Faedo C, et al: Metabolic effects of L-carnitine on type 2 diabetes mellitus: systematic review and meta-analysis. Exp Clin Endocrinol Diabetes 2013;121:234-238.

57 Lands B: Historical perspectives on the impact of n-3 and n-6 nutrients on health. Prog Lipid Res 2014;55:17-29.

58 Munro IA, Garg ML: Dietary supplementation with long chain omega-3 polyunsaturated fatty acids and weight loss in obese adults. Obes Res Clin Pract 2013; 7:e173-e181.

59 Munro IA, Garg ML: Dietary supplementation with n-3 PUFA does not promote weight loss when combined with a very-low-energy diet. Br J Nutr 2012;108:1466-1474.
60 Summers LK, Fielding BA, Bradshaw HA, et al: Substituting dietary saturated fat with polyunsaturated fat changes abdominal fat distribution and improves insulin sensitivity. Diabetologia 2002;45:369-377.

61 Crane FL: Biochemical functions of coenzyme Q10. J Am Coll Nutr 2001;20:591-598.

62 Lee YJ, Cho WJ, Kim JK, et al: Effects of coenzyme Q10 on arterial stiffness, metabolic parameters, and fatigue in obese subjects: a double-blind randomized controlled study. J Med Food 2011;14:386-390.

63 Kolahdouz MR, Hosseinzadeh-Attar MJ, Eshraghian MR, et al: The effect of coenzyme Q10 supplementation on metabolic status of type 2 diabetic patients. Minerva Gastroenterol Dietol 2013;59:231-236.

64 Hamilton SJ, Chew GT, Watts GF: Coenzyme Q10 improves endothelial dysfunction in statin-treated type 2 diabetic patients. Diabetes Care 2009;32:810-812.

65 Sohet FM, Delzenne NM: Is there a place for coenzyme Q in the management of metabolic disorders associated with obesity? Nutr Rev 2012;70:631-641

66 Gu L, Kelm MA, Hammerstone JF, et al: Liquid chromatographic/electrospray ionization mass spectrometric studies of proanthocyanidins in foods. J Mass Spectrom 2003;38:1272-1280.

67 Khan A, Safdar M, Ali Khan MM, et al: Cinnamon improves glucose and lipids of people with type 2 diabetes. Diabetes Care 2003;26: 3215-3218.

68 Mang B, Wolters M, Schmitt B, et al: Effects of a cinnamon extract on plasma glucose, $\mathrm{HbA}$, and serum lipids in diabetes mellitus type 2. Eur J Clin Invest 2006;36:340-344.

69 Lu T, Sheng H, Wu J, et al: Cinnamon extract improves fasting blood glucose and glycosylated hemoglobin level in Chinese patients with type 2 diabetes. Nutr Res 2012;32:408-412.

70 Roussel AM, Hininger I, Benaraba R, et al: Antioxidant effects of a cinnamon extract in people with impaired fasting glucose that are overweight or obese. J Am Coll Nutr 2009;28: 16-21.

71 Crawford P: Effectiveness of cinnamon for lowering hemoglobin $\mathrm{A} 1 \mathrm{C}$ in patients with type 2 diabetes: a randomized, controlled trial. J Am Board Fam Med 2009;22:507-512.

72 Vanschoonbeek K, Thomassen BJ, Senden JM, et al: Cinnamon supplementation does not improve glycemic control in postmenopausal type 2 diabetes patients. J Nutr 2006; 136:977-980.

73 Blevins SM, Leyva MJ, Brown J, et al: Effect of cinnamon on glucose and lipid levels in noninsulin-dependent type 2 diabetes. Diabetes Care 2007;30:2236-2237.

74 Woehrlin F, Fry H, Abraham K, et al: Quantification of flavoring constituents in cinnamon: high variation of coumarin in cassia bark from the German retail market and in authentic samples from Indonesia. J Agric Food Chem 2010;58:10568-10575. 
75 Ranasinghe P, Jayawardana R, Galappaththy $\mathrm{P}$, et al: Efficacy and safety of 'true' cinnamon (Cinnamomum zeylanicum) as a pharmaceutical agent in diabetes: a systematic review and meta-analysis. Diabet Med 2012;29:14801492.

76 All about green tea: major components and health benefits of green tea. http://www.itoen. co.jp/eng/allabout_greentea/components_ benefit.html. ITO EN, 2014

-77 Auvichayapat P, Prapochanung M, Tunkamnerdthai $\mathrm{O}$, et al: Effectiveness of green tea on weight reduction in obese Thais: a randomized, controlled trial. Physiol Behav 2008;93: 486-491.

78 Chantre P, Lairon D: Recent findings of green tea extract AR25 (Exolise) and its activity for the treatment of obesity. Phytomedicine 2002;9:3-8.

-79 Hsu CH, Liao YL, Lin SC, et al: Does supplementation with green tea extract improve insulin resistance in obese type 2 diabetics? A randomized, double-blind, and placebo-controlled clinical trial. Altern Med Rev 2011;16: 157-163.

80 Westerterp-Plantenga MS, Lejeune MP, Kovacs EM: Body weight loss and weight maintenance in relation to habitual caffeine intake and green tea supplementation. Obes Res 2005;13:1195-1204.

-81 Ammon HP: Biochemical mechanism of caffeine tolerance. Arch Pharm (Weinheim) 1991;324:261-267.

-82 Kang C, Lee H, Jung ES, et al: Saffron (Crocus sativus L.) increases glucose uptake and insulin sensitivity in muscle cells via multipathway mechanisms. Food Chem 2012;135: 2350-2358.
83 Vinson JA, Burnham BR, Nagendran MV: Randomized, double-blind, placebo-controlled, linear dose, crossover study to evaluate the efficacy and safety of a green coffee bean extract in overweight subjects. Diabetes Metab Syndr Obes 2012;5:21-27.

84 Onakpoya I, Terry R, Ernst E: The use of green coffee extract as a weight loss supplement: a systematic review and meta-analysis of randomised clinical trials. Gastroenterol Res Pract 2011;2011:382852.

85 Mancuso C, Santangelo R: Ferulic acid: pharmacological and toxicological aspects. Food Chem Toxicol 2014;65:185-195.

86 Karasawa K, Uzuhashi Y, Hirota M, et al: A matured fruit extract of date palm tree (Phoenix dactylifera L.) stimulates the cellular immune system in mice. J Agric Food Chem 2011;59:11287-11293.

87 Timmers S, Hesselink MK, Schrauwen P: Therapeutic potential of resveratrol in obesity and type 2 diabetes: new avenues for health benefits? Ann NY Acad Sci 2013;1290:83-89.

88 Brasnyo P, Molnar GA, Mohas M, et al: Resveratrol improves insulin sensitivity, reduces oxidative stress and activates the Akt pathway in type 2 diabetic patients. Br J Nutr 2011;106: 383-389.

89 Bhatt JK, Thomas S, Nanjan MJ: Resveratrol supplementation improves glycemic control in type 2 diabetes mellitus. Nutr Res 2012;32: 537-541.

90 Crandall JP, Oram V, Trandafirescu G, et al: Pilot study of resveratrol in older adults with impaired glucose tolerance. J Gerontol A Biol Sci Med Sci 2012;67:1307-1312.
91 Poulsen MM, Vestergaard PF, Clasen BF, et al: High-dose resveratrol supplementation in obese men: an investigator-initiated, randomized, placebo-controlled clinical trial of substrate metabolism, insulin sensitivity, and body composition. Diabetes 2013;62:11861195.

92 Yoshino J, Conte C, Fontana L, et al: Resveratrol supplementation does not improve metabolic function in nonobese women with normal glucose tolerance. Cell Metab 2012;16: 658-664.

93 Friedman M: Anticarcinogenic, cardioprotective, and other health benefits of tomato compounds lycopene, $\alpha$-tomatine, and tomatidine in pure form and in fresh and processed tomatoes. J Agric Food Chem 2013;61:95349550.

94 Coyne T, Ibiebele TI, Baade PD, et al: Diabetes mellitus and serum carotenoids: findings of a population-based study in Queensland, Australia. Am J Clin Nutr 2005;82:685-693.

95 Ghavipour M, Saedisomeolia A, Djalali M, et al: Tomato juice consumption reduces systemic inflammation in overweight and obese females. Br J Nutr 2013;109:2031-2035.

96 Upritchard JE, Sutherland WH, Mann JI: Effect of supplementation with tomato juice, vitamin E, and vitamin C on LDL oxidation and products of inflammatory activity in type 2 diabetes. Diabetes Care 2000;23:733-738.

97 Health Canada, Canadian Nutrient File. http://webprod3.hc-sc.gc.ca/cnf-fce/newNutrientSearch-nouvelleRechercheAliment. do?lang = eng/. 2012.

98 Linus Pauling Institute at Oregon State University, Micronutrient Information Center. http://lpi.oregonstate.edu/infocenter/contentnuts.html/. 2014. 